\title{
Experimental Recognition System for Dirty Eggshell by Using Image Analysis Technique
}

\author{
Abdullah Beyaz ${ }^{1, a, *}$, Serdar Özlï̈,b, Dilara Gerdan ${ }^{1, c}$ \\ ${ }^{1}$ Department of Agricultural Machinery and Technologies Engineering, Faculty of Agriculture, Ankara University, 06110 Ankara, Turkey \\ ${ }^{2}$ Department of Animal Science, Faculty of Agriculture, Ankara University, 06110 Ankara, Turkey \\ *Corresponding author
}

\begin{tabular}{|c|c|}
\hline A R T I C L E I N F O & A B S T R A C T \\
\hline $\begin{array}{l}\text { Keywords: } \\
\text { Egg } \\
\text { Image analysis } \\
\text { Dirty egg sorting }\end{array}$ & $\begin{array}{l}\text { The present study was focused on the design and implementation of an experimental recognition } \\
\text { system for dirty chicken eggshell by using an image analysis technique. Image analysis based } \\
\text { observation and evaluation techniques can be used efficiently and effectively for agricultural product } \\
\text { quality control. Dirt stains on eggs are the result of mainly by feces (black to light brown stains), uric } \\
\text { acid (white stains), yolk, and blood. The experimental system was used to obtain dark level images } \\
\text { of dirty stains of chicken eggs owing to feces. For this aim, the dirty chicken eggs which have dirty } \\
\text { parts were put under a webcam, and dirtiness degree was evaluated by using developed image } \\
\text { analysis software at the LabVIEW platform. For the experiment, } 100 \text { clean and } 100 \text { dirty eggs were } \\
\text { used to accurate the determination of dark stains. The results of the research showed that the designed } \\
\text { experimental system pointed an accuracy of } 99.8 \% \text { at painted grade eggs. On the other hand, the } \\
\text { accuracy of the differentiation of the dirt stains by feces was } 98.5 \% \text {. The developed system can be } \\
\text { upgraded for developing egg sorting machines by presence-absence of dirty stains in eggshell. }\end{array}$ \\
\hline
\end{tabular}

Food quality

LabVIEW

\section{Introduction}

Chicken eggs are one of the most valued food in the present life for people. In the world, billion tons of eggs $(1,386,697,882)$ were produced by different countries by the way Turkey's egg production was 18,097,605 tones in 2016 (Anonymous, 2018). Although the egg industry washes all table eggs sold to consumers, potential food safety concerns associated with the consumption of shell eggs exist. A variety of agents were identified, including protozoa, metazoan, fungi, viruses, and bacteria (Swayne et al., 1992). An estimated one in 20,000 eggs in the United States contain Salmonella Enteritis, and can cause illness if eaten raw or not thoroughly cooked ones are consumed (Anonymous, 1998). In most of the grading plants, the recognition of dirty eggs is still mainly performed by human graders (Mertens et al., 2011). A study has shown that human candling is not a very effective way of identifying dirty eggs when eggs are not washed. This is especially true for brown eggs which show a large variability in shell colour, making the contrasts created by dirt spots hardly visible. Egg handling companies have therefore developed and invested in new technologies to automate the visual inspection of eggs (Mertens et al., 2011).
According to Alberta Hatchery Association guidelines eggs are classified as: clean (no visible dirt); settable (dirt $<$ dimension size), dirty (dirt between a dimemension and quarter size) and unsettable (dirt > quarter size) (Schneider et al., 2016). On the other hand incubation of floor eggs or washed floor eggs resulted in lower hatchability compared to clean nest eggs, but that performance during the growout period was not affected (Van den Brand et al., 2016). However, dirty eggs can lead to high early mortality in young breeders. Improved breeder and hatchery management may be necessary and more effective than concentrating on the poults alone to tackle the problem (Hambrech et al., 2017).

Also this condition is the same on in broken and cracked egg shells. Some of the early studies on furnished cages also report an increase in broken and cracked shells (4-34\%) and a higher incidence of dirty eggs (7-26\%). This has usually been attributed to a large number of eggs being laid out of the nest and difficulties in maintaining cage hygiene (Rauch et al., 2002; Tauson et al., 2002; Michel et al. 2007). 
During the last two decades, lots of researchers have used computer vision to determine defects in eggs. Goodrum and Elster (1992), Patel et al., (1994a, b), Han and Feng (1994), Garcia-Alegre et al., (1997, 2000), Ribeiro et al., (2000), and Li et al., (2012) all presented the methods of determining the egg defects based on a vision system. The previous studies showed that the computer vision technique gives sometimes exciting results. Patel et al., (1998) worked on a study to determine blood spots, dirt stains, and cracks with the help of training neural networks by using colour histograms get from colour images. It resulted in an adequate performance for one defect but was not useful for evaluating eggs to determine more than one defect. From the past to now, there is no research presented quantified results on the differentiation of dirt stain recognition on eggs. Patel (1998) studied on an experimental system, and their experimental results showed that reduced the number of eggs requiring a human inspection from 95 to 44\%. Mertens et al., (2005) worked on the development of an offline computer vision system for the presence of different dirt stains on brown eggs: feces, uric acid, blood, and yolk stains. The accuracy of their system at different kinds of dirt stains was $91 \%$. The eggs with dark stains, $10.81 \%$ were wrongly detected for having bloodstains, and $33.33 \%$ of eggs with bloodstains were wrongly detected for having dark stains.

Moreover, Özkan et al., (2012) in their search aimed to find a quality control degree in the chicken egg and to calculate the volume of the egg, also to detect dirt on the egg surface. His achievement was $98 \%$ of success rate. Öztürk (2014) determined eggshell dirtiness, cracks, broken and bloodedness on chicken eggs by using image processing techniques. K-means classification technique, colour segmentation technique, the Sobel edge detection algorithm were applied on eggs. Also, Priyadumkol et al. (2017) worked on crack detection on unwashed eggs using image processing. They imply that their experiments were validated using 750 egg surface images with $94 \%$ accuracy and $1.67 \%$ false negatives.

The objective of the present work is to develope an upgrade system based on logical operators, using a basic algorithm which works free of variations in specific parameters, such as shell colour spaces and colour of the dirt stain for finding the efficiency of developed software dirtiness detection of dirty white eggs and to display results easily. In the light of literature, in this study, an experimental algorithm developed in the LabVIEW platform for eggshell feces detection.

\section{Materials and Methods}

\section{Hardware}

The designed dirty eggshell experimental quality control system based on real-time video measurements from dirt eggs with the help of a tripod supported USB webcam (Logitech C930e) and one computer (Asus UX490r). In the set-up, the webcam was placed $7 \mathrm{~cm}$ away from the egg and directed to the dirty place on the eggshell (Figure 1). The egg images were acquired in the RGB colour format (15 megapixels). Additionally, the video of eggshells were acquired in $1024 \times 768$ resolution by using webcam. The webcam was connected to the computer through a USB 3.0 connection.

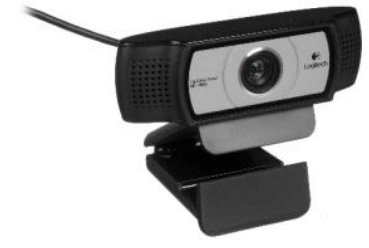

(a)

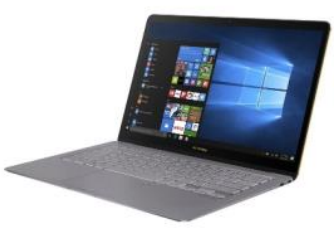

(b)
Figure 1. The equipment's used in the evaluations (Logitech C930e webcam (a) and Asus UX490r computer (b))

\section{Software}

The images were obtained by a webcam, and they were processed in LabVIEW Vision Assistant Software. Also, LabVIEW software was used for real-time video evaluations (Figure 2). There are three main steps in the software. First, the recognition of the egg to identify the region of interest for the recognition of dirt stains, second, the recognition and the third, classification of the dirt.

\section{Dirtiness Detection and Pretreatment}

A sorting software was developed to recognize dark stains reason for feces. Other stain types related to uric acid, egg yolk stains, and blood stains were not recognized by the software. Firstly, LabVIEW Vision Assistant Software was used for clean painted egg and dirty egg measurements as a primary measurement method for the calibration and reference measurements of the designed set-up from 100 clean painted and 100 dirty white eggs images (Figure 2, 3). To test the set-up is useful for dirt recognition in reality, a pretreatment experiment was carried out. For per treatment, one image was obtained per egg from a webcam which is placed perpendicular to the eggs at $7 \mathrm{~cm}$ distance. In the pretreatment process, it is mainly aimed that the image analysis system is working correctly or not. The output of the pretreatment process gave us a chance to understand that the image analysis of the spots and feces can also be detected and the system is working correctly.

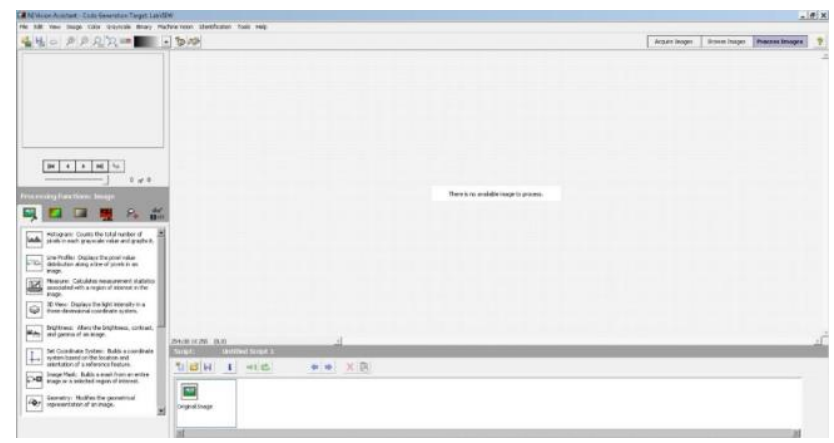

Figure 2. LabVIEW Vision Assistant software interface

Additionally, this pretreatment can be accepted as human candling because in this step both controls are done. It means, the spots and feces were controlled with image measurement like human vision, the first step was measurement control of digital images, so it can be accepted as the primary method before the video measurements. Because image measurements done in many studies in years and we know the achievement of image measurements. It means that it can be accepted as the primary control method. 


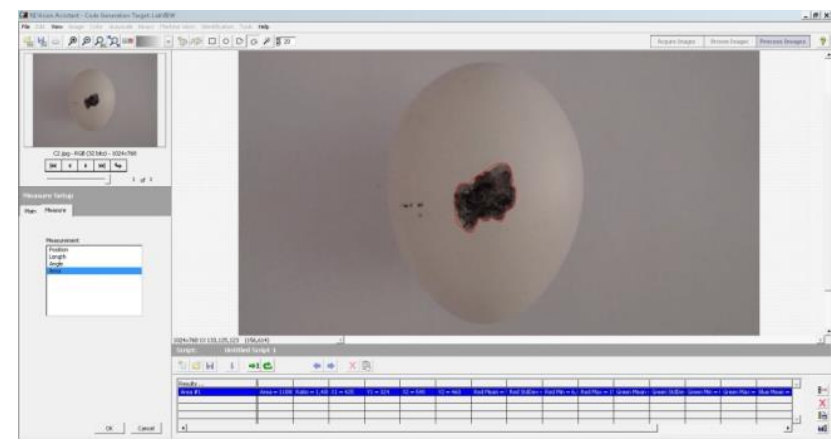

Figure 3. Primary dirtiness measurement process by using LabVIEW Vision Assistant software

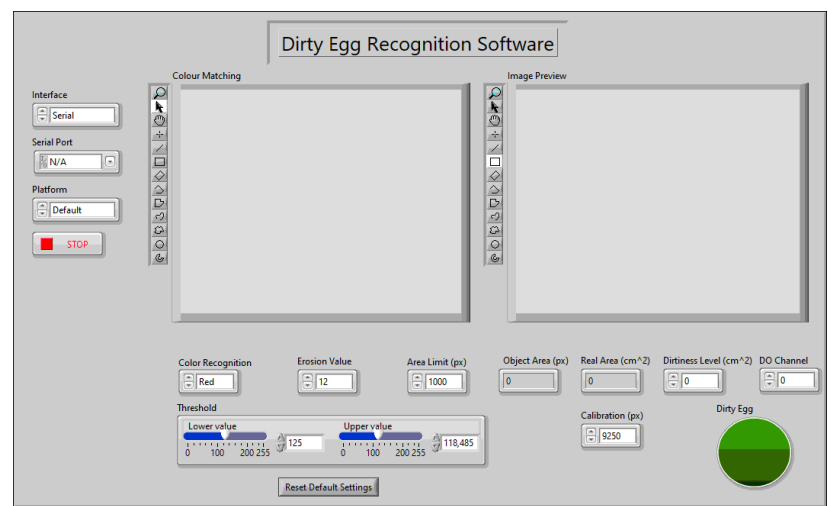

Figure 4. The front panel of dirty egg recognition software

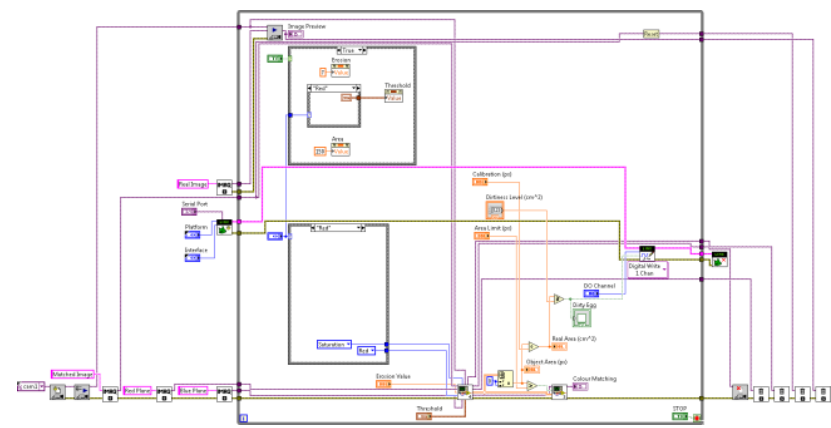

Figure 5. Block diagram of dirty egg recognition software

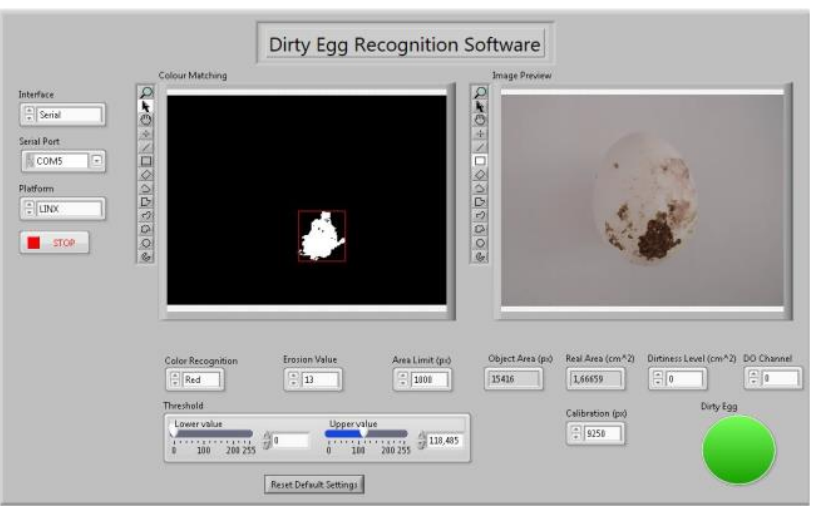

Figure 6. Live image on the front panel from the webcam

\section{Video Evaluation}

In the video evaluations, video measurements were done from continuous video streaming. Additionally, the webcam is placed perpendicular to the eggs at $7 \mathrm{~cm}$ distance like image measurement pre-treatment.

In the LabVIEW platform, a dirtiness recognition program was developed to detect feces stain. There are 1124 three main steps at this software. First, the recognition of the egg to define the interested region for the recognition of dirt stains, second is the sorting dirt and third is warning the user with a led placed on the front panel of the software according to the settled value. For this aim, first the software initialize the camera then create images for two planes as the analysis image and the preview image. After the colour range selection, it recognizes the dirt according to threshold, erosion and area limit values. It extracts the dirt area and shows it with bounding rectangle data. Then according to calibration value for a real-world measurement, it gives dirt area and warns the user about egg dirtiness. The software interface consists of various fields as shown in Figure 4.

Also, the block diagram has various parts for detecting the dirty eggshells according to user-defined colour space, area limit, erosion and threshold upper and lower values (Figure 5). Also the software allows to set freely dirtiness warning value according to user needs. In this application, a red colour channel with saturation value was used with 13 erosion and 0 minimum, 118,485 maximum threshold value. Calibration values evaluated as $9250 \mathrm{px}$ for $1 \mathrm{~cm}^{2}$. The dirtiness detached from live video of the eggshell which is display on the front panel of the developed software can be seen in Figure 6.

\section{Experimental Processes}

To test if the set-up is useful or not for dirt recognition in reality, an accuracy experiment was done. For this experiment, 200 Nick Chick eggs were used, 100 clean painted grade eggs and 100 dirty eggs. The sort of the eggs as clean or dirty was performed before handling by the producers at the egg production area. Then, the eggs with an open breakage were removed, and the feces stains on each dirty egg were observed. After that, each egg was placed in the test set-up and evaluated by the designed vision system (Figure 7). At last, the observations notes of the image and video measurement results of computer vision were compared.

\section{Experimental Processes}

Relationships between off-line calibration painted image measurement (CIM), and calibration painted realtime video measurement (CVM) were evaluated using general linear regression. Additionally, the graphics which contain the prediction equation and the regression value of the dirty egg image measurement (DIM) and dirty egg video measurements (DVM) of dirty eggs were evaluated. The regression lines were presented graphically as well as regression equations, and coefficients of determinations $\left(\mathrm{R}^{2}\right)$ were presented. The statistical analyses were performed using Minitab 14® software (Minitab, 2004).

\section{Results and Discussion}

The regression results of clean painted egg samples were presented in Figure 8 . The regression value was evaluated as $99.8 \%$ from the clean painted egg detection of experimental system. Also, the fact that the regression equation of the data was equal that $\mathrm{CIM}=0.04508+1.009$ CVM. According to the detection of dirty egg results, the algorithm has detected $98.5 \%$ correctly with the regression equation equal DIM $=0.09754+1.000 \mathrm{DVM}$, and $1.3 \%$ of 
samples have been detected as false (Figure 9). The $1.3 \%$ difference between clean painted egg results and dirty egg results comes from holes in dirtiness on the eggs, light intensity, and small displacement changes because of the diameter change of eggs. Patel et al. (1994b, 1998) find validation values for dirt detection of 83.33 and $85 \%$, respectively. Garcia-Allegre et al., (2000) reported an accuracy of $92 \%$ for dirty eggs and $82 \%$ for clean ones. Furthermore, Mertens et al. (2005) reported that vision system determined $100 \%$ of the dirty eggs and resulted with $2 \%$ of false rejects in the clean eggs, their total accuracy was $99 \%$ for the plain determination of dirt stains.

The detections were done immediately from webcam, but for this experimental application, the performance test done by using LabVIEW profile performance and memory tool. According to the results, total loop time was 218.8 millisecond, and overall memory usage was 19.35 kilobytes, but the system is not a full machine because of this reason there is no full machine performance or metrics. According to this data 16,453 egg can be sorted per hour if a prototype develops and other mechanic parts of the prototype system support. Additionally, as seen in figure 9, the success of the present system is $98.5 \%$, and the present system can recognize fecal dirt only, but the detection error rate is low enough in comparison with the other literature results because of the difference of the algorithms.

\section{Conclusion}

It is known that human handling can be applied with high performance, but we can say that this system could be used to recognize the dirty eggs owing to feces because it is fast as human perception. Only, the electronic, pneumatic or hydraulic part performances can make it slow according to their quality in that kind of machine. Additionally, egg processors have microbial problems as long as light dirty (or stained), dirty check and leaker are recognized as edible grades (Cantor et al., 1948). On the contrary, the dirty eggs are still detected by the workers in small and medium-sized enterprises/producers, according to the size of the operator in the dirt inspection process. Also, the image analysis-based systems can be use for many products to sort them according to their size and similarities. The developed experimental system technology for dirty stain detection in eggshell will have been encouraged in future studies. Additionally, it can be used as a measuring method to monitor dirty eggs in different housing systems and manufactures with such equipment. In other words, the developed system can be upgraded for developing egg sorting machines by presence-absence of dirty stains in eggshell.

Dirty eggs are still detected by the workers in small and medium-sized enterprises/producers. Also, the image analysis-based systems were used for many products to sort them by using different software solutions which have different performances. In this study, the developed experimental system technology for dirty stain detection in the eggshell is a new approach which uses the power of LabVIEW development platform alternative to Matlab and other software. Additionally, it can be used as a measuring method to monitor dirty eggs in different housing systems for manufacturers with such equipment. In other words, the developed system can be upgraded for developing egg sorting machines by presence-absence of dirty stains in eggshell, and we can say that this system could be used to recognize the dirty eggs owing to feces because it is fast as human perception.

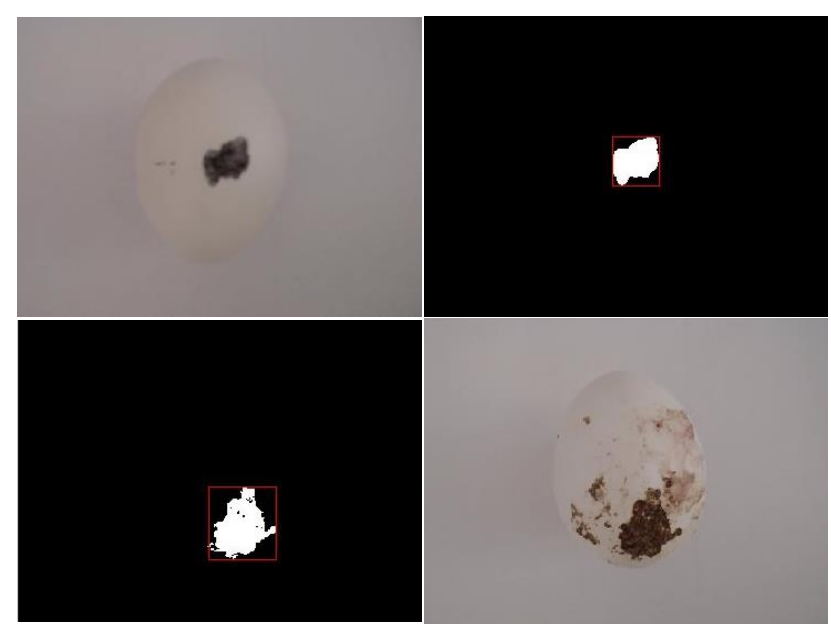

Figure 7. Video measurements of clean painted and dirty eggs

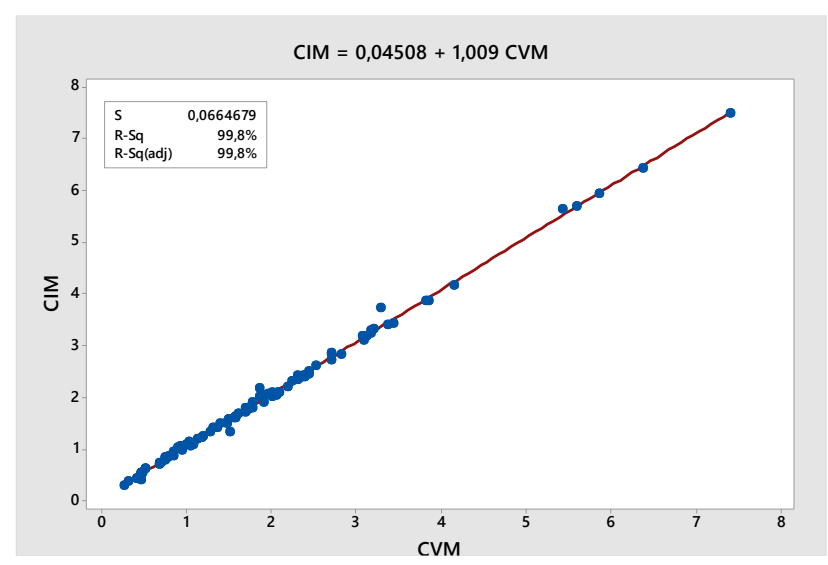

Figure 8. LabVIEW Vision Assistant - Image and Video measurement regression value and estimation equation which belongs to clean painted egg samples

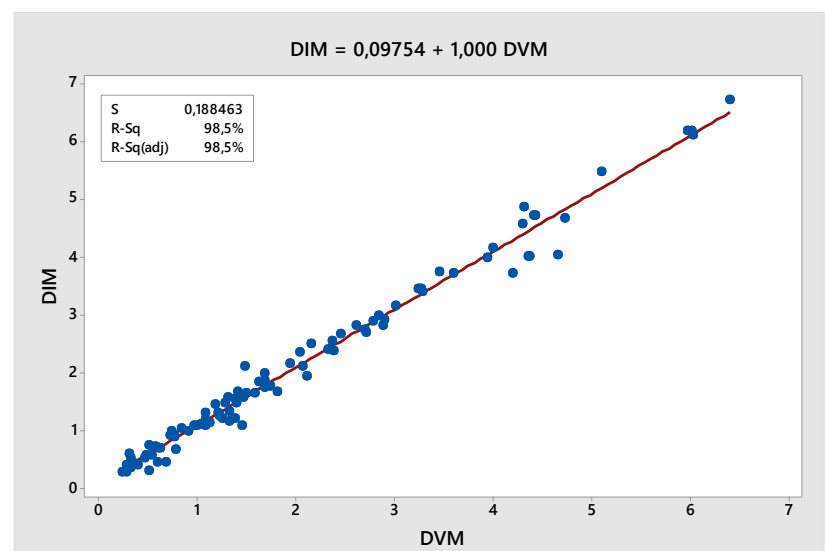

Figure 9. LabVIEW Vision Assistant - Image and Video measurement regression value and estimation equation which belongs to dirty white egg samples

\section{References}

Anonymous, 1998. Salmonella Enteritidis risk assessment: shell eggs and egg products, final report.U.S. Department of Agriculture, Food Safety and Inspection Service, Washington, D. C. 
Anonymous, 2018. Available from: http://www.fao.org/faostat/ en/\#data/QL. (14.09.2018).

Cantor A, McFarlane VH. 1948. Salmonella organisms on and in chicken eggs. Poult. Sci., 27:350-355.

Elster RT, Goodrum JW. 1991. Detection of cracks in eggs using machine vision. Trans. ASAE, 30:307-312.

Garcia-Alegre MC, Enciso J, Ribeiro A, Guinea D. 1997. Towards an automatic visual inspection of eggshell defects. Proc. Int. Workshop on Robotics and Automated Machinery for Bio-Productions, Gandia, Spain, 51-66.

Garcia-Alegre MC, Ribeiro A, Guinea D, Cristobal G. 2000. Colour index analysis for automatic detection of eggshell defects. Proc. SPIE, 3966:380-387.

Goodrum JW, Elster RT. 1992. Machine vision for crack detection in rotating eggs. Trans. ASAE., 35: 1323-1328.

Hambrech E, Teirlynck E, Page G, Leek A. 2017. Turkey production without antibiotics Proc. of the 11th. Turkey Science and Production Conference, Chester, UK.

Han YJ, Feng Y. 1994. Egg shell inspection using global image analysis. Appl. Eng. Agric., 10:109-114.

Li Y, Dhakal S, Peng Y. 2012. A machine vision system for identification of micro-crack in egg shell. J. Food Eng., 109:127-134.

Mertens K, De Ketelaere B, Kamers B, Bamelis FR, Kemps BJ, Verhoelst EM, De Baerdemaeker JG, Decuypere EM. 2005. Dirt detection on brown eggs by means of colour computer vision. Poult. Sci., 84: 1653-1659.

Mertens K, Kemps B, Perianu C, De Baerdemaeker J, Decuypere E, De Ketelaere B. 2011. Advances in defect detection, quality assessment and automated sorting and grading. In Improving the safety and quality of eggs and egg products. Ed. Yves Nys, Maureen Bain and Filip Van Immerseel. Voodhead Publishing Ltd., Cambridge, U.K.

Michel V, Arnould C, Mirabito L, Guemene D. 2007. Systeme de production et bien-etre en elevage de poules pondeuses, Inra Prod. Anim., 20: 47-52.

Minitab Inc. 2004. Minitab Release Version 14, Minitab Inc., State Collage, PA.

Özkan H. 2012. A real-time quality control application for animal production by image processing. Master Thesis, Department of Electronic and Computer Education, Selçuk University.
Öztürk N. 2014. Eggshell defects detection on white eggs using image processing techniques. Karadeniz Technical University, Institute of Science, Department of Electrical and Electronics Engineering, Master Thesis, Trabzon, Turkey.

Patel VC, McClendon RW, Goodrum JW. 1994a. Crack detection in eggs using computer vision and neural networks. Artif. Intell. Appl., 8: 21-31.

Patel VC, McClendon RW, Goodrum JW. 1994b. Detection of blood spots and dirt stains in eggs using computer vision and neural networks. Report No. 94-G-034., Proc. Int. Conf. Agric. Eng., Milan, Italy, 833-834.

Patel VC, McClendon RW, Goodrum JW. 1998. Development and evaluation of an expert system for egg sorting. Comput Electron Agric., 20 (1998): 97-116.

Priyadumkol J, Kittichaikarn C, Thainimit S. 2017. Crack detection on unwashed eggs using image processing. J. Food Eng., 209 (2017): 76-82.

Rauch HW, Buchenauer D, Hartung J, Hinrichs P. 2002. Experiences from various furnished cage models, 11th European Poultry Conference, Bremen, Germany.

Ribeiro A, Garcia-Alegre MC, Guinea D, Cristobal G. 2000. Automatic rules generation by G.A. for eggshell defect classification. Eur. Congr. Comput. Methods Appl. Sci. Eng., Barcelona, Spain.

Schneider B, Josephson J, Gensler G, Robinson N, Carney V. 2016. Supporting practice change on-farm: modifying hatching egg washing practices. 2016 Int. Poult. Sci. Cong., Atlanta, Georgia, USA.

Swayne DE, Bermudez AJ, Sagartz JE, Eaton KA, Monfort JD, Stoutenburg JW, Hayes JR. 1992. Association of cecal spirochetes with pasty vents and dirty eggshells in layers. Avian Diseases, 776-781.

Tauson R, Holm KE, Wall H. 2002. Experiences from various furnished cage models in Sweden. 11th European Poultry Conference, Bremen, Germany.

Van den Brand H, Sosef MP, Lourens A, Van Harn J. 2016. Effects of flor eggs on hatchability and later life performance in broiler chickens. Poult. Sci., 95: 1025-1032. 